\title{
Radiative decays of $\phi$-meson and nature of light scalar resonances
}

\author{
N.N. Achasov \\ Laboratory of Theoretical Physics, Sobolev Institute for Mathematics, Novosibirsk, 630090, Russia
}

(November 10, 2018)

\begin{abstract}
Based on gauge invariance, we show that the new threshold phenomenon is discovered in the $\phi$ radiative decays $\phi \rightarrow \gamma a_{0} \rightarrow \gamma \pi^{0} \eta$ and $\phi \rightarrow \gamma f_{0} \rightarrow \gamma \pi^{0} \pi^{0}$. This enables to conclude that production of the lightest scalar mesons $a_{0}(980)$ and $f_{0}(980)$ in these decays is caused by the four-quark transitions, resulting in strong restrictions on the large $N_{C}$ expansions of the decay amplitudes. The analysis shows that these constraints give new evidences in favor of the four-quark nature of $a_{0}(980)$ and $f_{0}(980)$ mesons.
\end{abstract}

PACS number(s): 12.39.-x, 13.40.Hq, 13.65.+i

Typeset using REVTEX 
The discovered more than thirty years ago the lightest scalar mesons $a_{0}(980)$ and $f_{0}(980)$ became the hard problem for the naive quark-antiquark $(q \bar{q})$ model from the outset. Really, on the one hand the almost exact degeneration of the masses of the isovector $a_{0}(980)$ and isoscalar $f_{0}(980)$ states revealed seemingly the structure similar to the structure of the vector $\rho$ and $\omega$ mesons, and on the other hand the strong coupling of $f_{0}(980)$ with the $K \bar{K}$ channel pointed unambiguously to a considerable part of the strange quark pair $s \bar{s}$ in the wave function of $f_{0}(980)$.

In 1977 R.L. Jaffe noted that in the MIT bag model, which incorporates confinement phenomenologically, there are light four-quark scalar states [1]. He suggested that $a_{0}(980)$ and $f_{0}(980)$ might be these states with symbolic structures $a_{0}^{0}(980)=(u s \bar{u} \bar{s}-d s \bar{d} \bar{s}) / \sqrt{2}$ and $f_{0}(980)=(u s \bar{u} \bar{s}+d s \bar{d} \bar{s}) / \sqrt{2}$. From that time $a_{0}(980)$ and $f_{0}(980)$ resonances came into beloved children of the light quark spectroscopy, see, for example, reviews [2. 3].

Ten years later we showed 四 that the study of the radiative decays $\phi \rightarrow \gamma a_{0} \rightarrow \gamma \pi \eta$ and $\phi \rightarrow \gamma f_{0} \rightarrow \gamma \pi \pi$ can shed light on the problem of $a_{0}(980)$ and $f_{0}(980)$ mesons. Over the next ten years the question was considered from different points of view [5 8 ].

Now these decays have been studied not only theoretically but also experimentally, so that the time is ripe to sum up.

Present time data have already been obtained from Novosibirsk with the detectors SND [9.10] and CMD-2 [11], which give the following branching ratios: $B R\left(\phi \rightarrow \gamma \pi^{0} \eta\right)=$ $(0.88 \pm 0.14 \pm 0.09) \cdot 10^{-4}$ [9] (2000), $B R\left(\phi \rightarrow \gamma \pi^{0} \pi^{0}\right)=(1.221 \pm 0.098 \pm 0.061) \cdot 10^{-4}$ [10 (2000) and $B R\left(\phi \rightarrow \gamma \pi^{0} \eta\right)=(0.9 \pm 0.24 \pm 0.1) \cdot 10^{-4}, B R\left(\phi \rightarrow \gamma \pi^{0} \pi^{0}\right)=(0.92 \pm 0.08 \pm 0.06) \cdot 10^{-4}$ [11]. DA $\Phi N E$ also confirms the Novosibirsk results [12].

These data give evidence in favor of the four-quark $\left(q^{2} \bar{q}^{2}\right)$ [1],3], 13, [14] nature of $a_{0}(980)$ and $f_{0}(980)$ mesons [4, 6, 15]. Note that the isovector $a_{0}(980)$ meson is produced in the radiative $\phi$ meson decay as intensively as the well-studied $\eta^{\prime}(958)$ meson containing $\approx 66 \%$ of $s \bar{s}$, responsible for the decay . It is a clear qualitative argument for the presence of the $s \bar{s}$ pair in the isovector $a_{0}(980)$ state, i.e., for its four-quark nature.

Since the one-loop model $\phi \rightarrow K^{+} K^{-} \rightarrow \gamma a_{0}$ and $\phi \rightarrow K^{+} K^{-} \rightarrow \gamma f_{0}$, suggested at 
basing the experimental investigations [4], was used in the data treatment from the outset, the question on the mechanism of the scalar meson production in the $\phi$ radiative decays was put into the shade. We show below that the present data give the conclusive arguments in favor of the $K^{+} K^{-}$loop mechanism of $a_{0}(980)$ and $f_{0}(980)$ mesons production in the $\phi$ radiative decays. The knowledge of this mechanism allows to conclude that the production of $a_{0}(980)$ and $f_{0}(980)$ in the $\phi$ radiative decays is caused by the four-quark transitions. This constrains the large $N_{C}$ expansions of the decay amplitudes and gives new impressive evidences in favor of the four-quark nature of $a_{0}(980)$ and $f_{0}(980)$.

In Figs. 11 and 2 are shown the SND data on $\phi \rightarrow \gamma \pi^{0} \eta$ [9] (2000) and $\phi \rightarrow \gamma \pi^{0} \pi^{0}$ [10] (2000). The data are described in the following model $\phi \rightarrow\left(\gamma a_{0}+\pi^{0} \rho\right) \rightarrow \gamma \pi^{0} \eta$ and $\phi \rightarrow\left(\gamma f_{0}+\pi^{0} \rho\right) \rightarrow \gamma \pi^{0} \pi^{0}$, see details in Ref. [15]. It is important for us now nothing but the fact that the $\phi \rightarrow \gamma a_{0} \rightarrow \gamma \pi^{0} \eta$ process dominates everywhere over the region of the $\pi^{0} \eta$ invariant mass $m_{\pi^{0} \eta}=m$ and the $\phi \rightarrow \gamma f_{0} \rightarrow \gamma \pi^{0} \pi^{0}$ process dominates in the resonance region of the $\pi^{0} \pi^{0}$ system, the $\pi^{0} \pi^{0}$ invariant mass $m_{\pi^{0} \pi^{0}}=m>670 \mathrm{MeV}$ [16].

The resonance mass spectrum is of the form [17

$$
\begin{aligned}
& S_{R}(m)=d \Gamma(\phi \rightarrow \gamma R \rightarrow \gamma a b, m) / d m \\
& =\frac{2}{\pi} \frac{m^{2} \Gamma(\phi \rightarrow \gamma R, m) \Gamma(R \rightarrow a b, m)}{\left|D_{R}(m)\right|^{2}}=\frac{4\left|g_{R}(m)\right|^{2} \omega(m) p_{a b}(m)}{3(4 \pi)^{3} m_{\phi}^{2}}\left|\frac{g_{R a b}}{D_{R}(m)}\right|^{2},
\end{aligned}
$$

where $R=a_{0}$ or $f_{0}$ and $a b=\pi^{0} \eta$ or $\pi^{0} \pi^{0}$ respectively, $\omega(m)=\left(m_{\phi}^{2}-m^{2}\right) / 2 m_{\phi}$ is the photon energy in the $\phi$ meson rest frame, $p_{a b}(m)$ is the modulus of the $a$ or $b$ particle momentum in the $a$ and $b$ mass center frame, $g_{R a b}$ is the coupling constant, $g_{f_{0} \pi^{0} \pi^{0}}=g_{f_{0} \pi^{+} \pi^{-}} / \sqrt{2}$, $D_{R}(m)$ is the $R$ resonance propagator the form of which everywhere over the $m$ region can be find in $4,18,19, g_{R}(m)$ is the invariant amplitude that describes the vertex of the $\phi(p) \rightarrow \gamma(k) R(q)$ transition with $q^{2}=m^{2}$. This is precisely the function which is the subject of our investigation.

By gauge invariance, the transition amplitude is proportional to the electromagnetic field strength tensor $F_{\mu \nu}$ (in our case to the electric field in the $\phi$ meson rest frame):

$$
A[\phi(p) \rightarrow \gamma(k) R(q)]=G_{R}(m)\left[p_{\mu} e_{\nu}(\phi)-p_{\nu} e_{\mu}(\phi)\right]\left[k_{\mu} e_{\nu}(\gamma)-k_{\nu} e_{\mu}(\gamma)\right]
$$


where $e(\phi)$ and $e(\gamma)$ are the $\phi$ meson and $\gamma$ quantum polarization four-vectors, $G_{R}(m)$ is the invariant amplitude free from kinematical singularities. Since there are no charge particles or particles with magnetic moments in the process, there is no pole in $G_{R}(m)$. Consequently, the function

$$
g_{R}(m)=-2(p k) G_{R}(m)=-2 \omega(m) m_{\phi} G_{R}(m)
$$

is proportional to $\omega(m)$ (at least!) in the soft photon region.

To describe the experimental spectra in Figs. 道 and 2, the function $\left|g_{R}(m)\right|^{2}$ should be smooth (almost constant) in the range $m \leq 0.99 \mathrm{GeV}$, see Eq. (1). Stopping the function $(\omega(m))^{2}$ at $\omega(990 \mathrm{MeV})=29 \mathrm{MeV}$ with the help of the form-factor $1 /\left[1+(R \omega(m))^{2}\right]$ requires $R \approx 100 \mathrm{GeV}^{-1}$. It seems to be incredible to explain the formation of such a huge radius in hadron physics. Based on the large, by hadron physics standard, $R \approx 10 \mathrm{GeV}^{-1}$, one can obtain an effective maximum of the mass spectrum under discussion only near 900 $\mathrm{MeV}$. To exemplify this trouble let us consider the contribution of the isolated $\mathrm{R}$ resonance: $g_{R}(m)=-2 \omega(m) m_{\phi} G_{R}\left(m_{R}\right)$. Let also the mass and the width of the $\mathrm{R}$ resonance equal $980 \mathrm{MeV}$ and $60 \mathrm{MeV}$, then $S_{R}(920 \mathrm{MeV}): S_{R}(950 \mathrm{MeV}): S_{R}(970 \mathrm{MeV}): S_{R}(980 \mathrm{MeV})=$ $3: 2.7: 1.8: 1$.

So stopping the $g_{R}(m)$ function is the crucial point in understanding the mechanism of the production of $a_{0}(980)$ and $f_{0}(980)$ resonances in the $\phi$ radiative decays.

The $K^{+} K^{-}$loop model $\phi \rightarrow K^{+} K^{-} \rightarrow \gamma R$ [4] solves this problem in the elegant way: the fine threshold phenomenon is discovered, see Fig. 36, where the universal in $K^{+} K^{-}$loop model function $|g(m)|^{2}=\left|g_{R}(m) / g_{\text {Rab }}\right|^{2}$ is shown [20].

To demonstrate the threshold character of this effect we present Fig. 4 and Fig. 5 in which the function $|g(m)|^{2}$ is shown in the case of $K^{+}$meson mass is $25 \mathrm{MeV}$ and $50 \mathrm{MeV}$ less than in reality.

One can see from Figs. 因 and 0 that the function $|g(m)|^{2}$ is suppressed by the $(\omega(m))^{2}$ law in the region 950-1020 MeV and 900-1020 Mev respectively [21].

In the mass spectrum this suppression is increased by one more power of $\omega(m)$, see Eq. 
(四), so that we cannot see the resonance in the region $980-995 \mathrm{MeV}$. The maximum in the spectrum is effectively shifted to the region $935-950 \mathrm{MeV}$ and $880-900 \mathrm{MeV}$ respectively.

In truth this means that $a_{0}(980)$ and $f_{0}(980)$ resonances are seen in the radiative decays of $\phi$ meson owing to the $K^{+} K^{-}$intermediate state, otherwise the maxima in the spectra would be shifted to $900 \mathrm{MeV}$.

So the mechanism of production of $a_{0}(980)$ and $f_{0}(980)$ mesons in the $\phi$ radiative decays is established.

Both the real part and the imaginary one of the $\phi \rightarrow \gamma R$ amplitude are caused by the $K^{+} K^{-}$intermediate state. The imaginary part is caused by the real $K^{+} K^{-}$intermediate state while the real part is caused by the virtual compact $K^{+} K^{-}$intermediate state, i.e., we are dealing here with the four-quark transition [22] . Needless to say, radiative four-quark transitions can happen between two $q \bar{q}$ states as well as between $q \bar{q}$ and $q^{2} \bar{q}^{2}$ states but their intensities depend strongly on a type of the transitions. A radiative four-quark transition between two $q \bar{q}$ states requires creation and annihilation of an additional $q \bar{q}$ pair, i.e., such a transition is forbidden according to the Okuba-Zweig-Izuka (OZI) rule, while a radiative four-quark transition between $q \bar{q}$ and $q^{2} \bar{q}^{2}$ states requires only creation of an additional $q \bar{q}$ pair, i.e., such a transition is allowed according to the OZI rule.

Let us consider this problem from the large $N_{C}$ expansion standpoint. Let us begin with the $q \bar{q}$ model.

In the two-quark model $a_{0}^{0}(980)=(u \bar{u}-d \bar{d}) / \sqrt{2}$, the large $N_{C}$ expansion of the $\phi=$ $s \bar{s} \rightarrow \gamma a_{0}(980)$ amplitude starts with the OZI forbidden transition of the $1 / N_{C}$ order $(s \bar{s}$ annihilation and $u \bar{u}, d \bar{d}$ creation). But its weight is bound to be small, because this term does not contain the $K^{+} K^{-}$intermediate state emerging only in the next to leading term of the $\left(1 / N_{C}\right)^{2}(!)$ order for creation and annihilation of additional $q \bar{q}$ pairs. It will be recalled that $\phi \rightarrow \gamma \eta^{\prime}(958)$ transition does not require creation of an additional $q \bar{q}$ pair at all (the OZI superallowed transition) and has the $\left(N_{C}\right)^{0}$ order.

In the two-quark model $f_{0}(980)=(u \bar{u}+d \bar{d}) / \sqrt{2}$, which involves the $a_{0}-f_{0}$ mass degeneration, the large $N_{C}$ expansion of the $\phi=s \bar{s} \rightarrow \gamma f_{0}(980)$ amplitude starts also with the OZI 
forbidden transition of the $1 / N_{C}$ order, whose weight also is bound to be small, because this term does not contain the $K^{+} K^{-}$intermediate state emerging only in the next to leading term of the $\left(1 / N_{C}\right)^{2}$ order.

In the two-quark model $f_{0}(980)=s \bar{s}$, which has the serious trouble with the $a_{0}-f_{0}$ mass degeneration, the $\left(N_{C}\right)^{0}$ order transition without creation of an additional $q \bar{q}$ pair ( similar in this regard to $\left.\phi \rightarrow \gamma \eta^{\prime}(958)\right)$ is bound to have a small weight in the large $N_{C}$ expansion of the $\phi=s \bar{s} \rightarrow \gamma f_{0}(980)$ amplitude, because this term does not contain the $K^{+} K^{-}$intermediate state emerging only in the next to leading term of the $1 / N_{C}$ order, i.e., in the OZI forbidden transition. Emphasize that the mechanism without creation and annihilation of the additional $u \bar{u}$ pair cannot explain the $S_{f_{0}}(m)$ spectrum because it does not contain the $K^{+} K^{-}$intermediate state!

But if $a_{0}^{0}(980)$ and $f_{0}(980)$ mesons are compact $K \bar{K}$ states $(u \bar{s} s \bar{u}-d \bar{s} s \bar{d}) / \sqrt{2}$ and $(u \bar{s} s \bar{u}+$ $d \bar{s} s \bar{d}) / \sqrt{2}$ respectively, i.e., four-quark states similar to states of the MIT-bag model [23], the large $N_{C}$ expansions of the $\phi=s \bar{s} \rightarrow \gamma a_{0}(980)$ and $\phi=s \bar{s} \rightarrow \gamma f_{0}(980)$ amplitudes start with the OZI allowed transitions of the $\left(1 / N_{C}\right)^{-1 / 2}$ order, which require only creation the additional $u \bar{u}$ pair for the $K^{+} K^{-}$intermediate state. It will be recalled that a OZI allowed hadronic decay amplitude, for example, the $\rho \rightarrow \pi \pi$ amplitude, has the $\left(1 / N_{C}\right)^{-1 / 2}$ order.

In summary the fine threshold phenomenon is discovered, which is to say that the $K^{+} K^{-}$ loop mechanism of the $a_{0}(980)$ and $f_{0}(980)$ scalar meson production in the $\phi$ radiative decays is established at a physical level of proof. The case is rarest in hadron physics. This production mechanism is the four-quark transition what constrains the large $N_{C}$ expansion of the $\phi \rightarrow \gamma a_{0}(980)$ and $\phi \rightarrow \gamma f_{0}(980)$ amplitudes and gives the new strong (if not crucial) evidences in favor of the four-quark nature of $a_{0}(980)$ and $f_{0}(980)$ mesons. 


\section{REFERENCES}

[1] R.L. Jaffe, Phys. Rev. D 15, 267, 281 (1977).

[2] L. Montanet, Rep. Prog. Phys. 46, 337 (1983); F.E. Close, Rep. Prog. Phys. 51, 833 (1988); L.G. Landsberg, Usp. Fiz. Nauk. 160, 3 (1990) [Sov. Phys. Usp. 33, 1 (1990)]; M.R. Pennington, HADRON '95, Proceedings of the 6h International Conference on Hadron Spectroscopy, Manchester, UK, 10th-14th July 1995, Eds. M.C. Birse, G.D. Lafferty, and J.A. McGovern, page 3, World Scientific, Singapore; T. Barnes, HADRON SPECTROSCOPY, VII International Conference, Upton, NY August 1997, Eds. S.U. Chung, H.J. Willutzki, AIP CONFERENCE PROCEEDINGS 432, page 3; C. Amsler, Rev.Mod.Phys. 70, 1293 (1998); Muneyuki Ishida, Shin Ishida, and Taku Ishida, Prog.Theor.Phys. 99, 1031 (1998); R. Delbourgo and M.D. Scadron, Int. J. Mod. Phys. A 13, 657 (1998); S. Godfrey and J. Napolitano, Rev. Mod. Phys. 71, 1411 (1999); P. Minkowski and W. Ochs, Eur. Phys. J. C 9, 283 (1999); S. Spanier and N.A. Törnqvist, Eur. Phys. J. C 15, 437 (2000); E. van Beveren, hep-ph/0201006.

[3] N.N. Achasov, S.A. Devyanin and G.N. Shestakov, Usp. Fiz. Nauk. 142, 361 (1984) [Sov. Phys. Usp. 27, 161 (1984)]; N.N. Achasov and G.N. Shestakov, Usp. Fiz. Nauk. 161 (6), 53 (1991)[Sov. Phys. Usp. 34 (6), 471 (1991)]; N.N. Achasov, Nucl. Phys. B (Proc. Suppl.) 21, 189 (1991); N.N. Achasov, Usp. Fiz. Nauk. 168, 1257 (1998) [ Phys. Usp. 41, 1149 (1998)]; N.N. Achasov, Nucl. Phys. A 675, 279c (2000).

[4] N.N. Achasov and V.N. Ivanchenko, Nucl. Phys. B 315, 465 (1989); Preprint INP 87-129 (1987) Novosibirsk.

[5] A. Bramon, A. Grau, G. Pancheri, Phys.Lett. B 289, 97 (1992); F.E. Close, N. Isgur, and S. Kumano, Nucl. Phys. B 389, 513 (1993); E. Marco, S. Hirenzaki, E. Oset, and H. Toki, Phys. Lett. B 470, 20 (1999); J.L. Lucio, M. Napsuciale, Contribution to 3rd Workshop on Physics and Detectors for DAPHNE (DAPHNE 99), Frascati, Italy, 1999.

[6] N.N. Achasov and V.V. Gubin, Phys. Rev. D 56, 4084 (1997). 
[7] N.N. Achasov, V.V. Gubin, and V.I. Shevchenko, Phys. Rev. D 56, 203 (1997).

[8] N.N. Achasov, V.V. Gubin, and E.P. Solodov, Phys. Rev. D 55, 2672 (1997); N.N. Achasov and V.V. Gubin, Phys. Rev. D 57, 1987 (1998).

[9] M.N. Achasov et al., Phys. Lett. B 438, 441 (1998); 479, 53 (2000).

[10] M.N. Achasov et al., Phys. Lett. B 440, 442 (1998); 485, 349 (2000).

[11] R.R. Akhmetshin et al., Phys. Lett. B 462, 380 (1999).

[12] Barbara Valeriani, Parallel session talk at HADRON 2001, Proceedings.

[13] O. Black, A. Fariborz, F. Sannino, and J. Schechter, Phys.Rev. D 59, 074026 (1999).

[14] HADRON 2002, Proceedings: J. Schechter (Plenary session talk), hep-ph/0110356; S.F. Tuan ( Parallel session talk), hep-ph/0109191; T. Teshima (Parallel session talk), hepph/0105107.

[15] N.N. Achasov and V.V. Gubin, Phys. Rev. D 63, 094007 (2001).

[16] The difference from the experimental data, observed in the region $m_{\pi \pi}<670 \mathrm{MeV}$, is due to the fact that in the experimental processing of the $e^{+} e^{-} \rightarrow \gamma \pi^{0} \pi^{0}$ events the background events $e^{+} e^{-} \rightarrow \omega \pi^{0} \rightarrow \gamma \pi^{0} \pi^{0}$ are excluded with the help of the invariant mass cutting, in so doing the part of the $e^{+} e^{-} \rightarrow \phi \rightarrow \rho \pi^{0} \rightarrow \gamma \pi^{0} \pi^{0}$ events is excluded as well.

[17] Notice that in Ref. [15] we took into account the mixing of $f_{0}(980)$ meson with another scalar isoscalar resonance, see also Ref. [6], but such a complication is not essential for the present investigation.

[18] N.N. Achasov, S.A. Devyanin, and G.N. Shestakov, Yad. Fiz. 32, 1098 (1980)[Sov. J. Nucl. Phys. 32, 566 (1980)].

[19] N.N. Achasov and V.V. Gubin, Phys. Rev. D 64, 094016 (2001). 
[20] The forms of $g_{R}(m)$ and $g(m)=g_{R}(m) / g_{R a b}$ everywhere over the $m$ region are in Refs. [4] and [19] respectively.

[21] The actual absence of a background at a soft photon energy region $\omega(m)>112 \mathrm{MeV}$ $(m>900 \mathrm{MeV})$ owes to gauge invariance also.

[22] It will be recalled that the imaginary part of every hadronic amplitude describes a multi-quark transition.

[23] In the case of the $K \bar{K}$ bound states with the binding energy close to $20 \mathrm{MeV}$, i.e.,in the extended molecule case, the contribution of the virtual intermediate $K^{+} K^{-}$states in the $K^{+} K^{-}$loop is suppressed by the momentum distribution in the molecule, and the real part of the loop amplitudes are negligible [7]. It leads to the branching ratios much less than the experimental ones. In addition, the $S_{R}(m)$ spectra in the $K \bar{K}$ molecule case are much narrower than the experimental ones, see the behavior of the imaginary part contribution in Fig. 3 . 


\section{FIGURES}

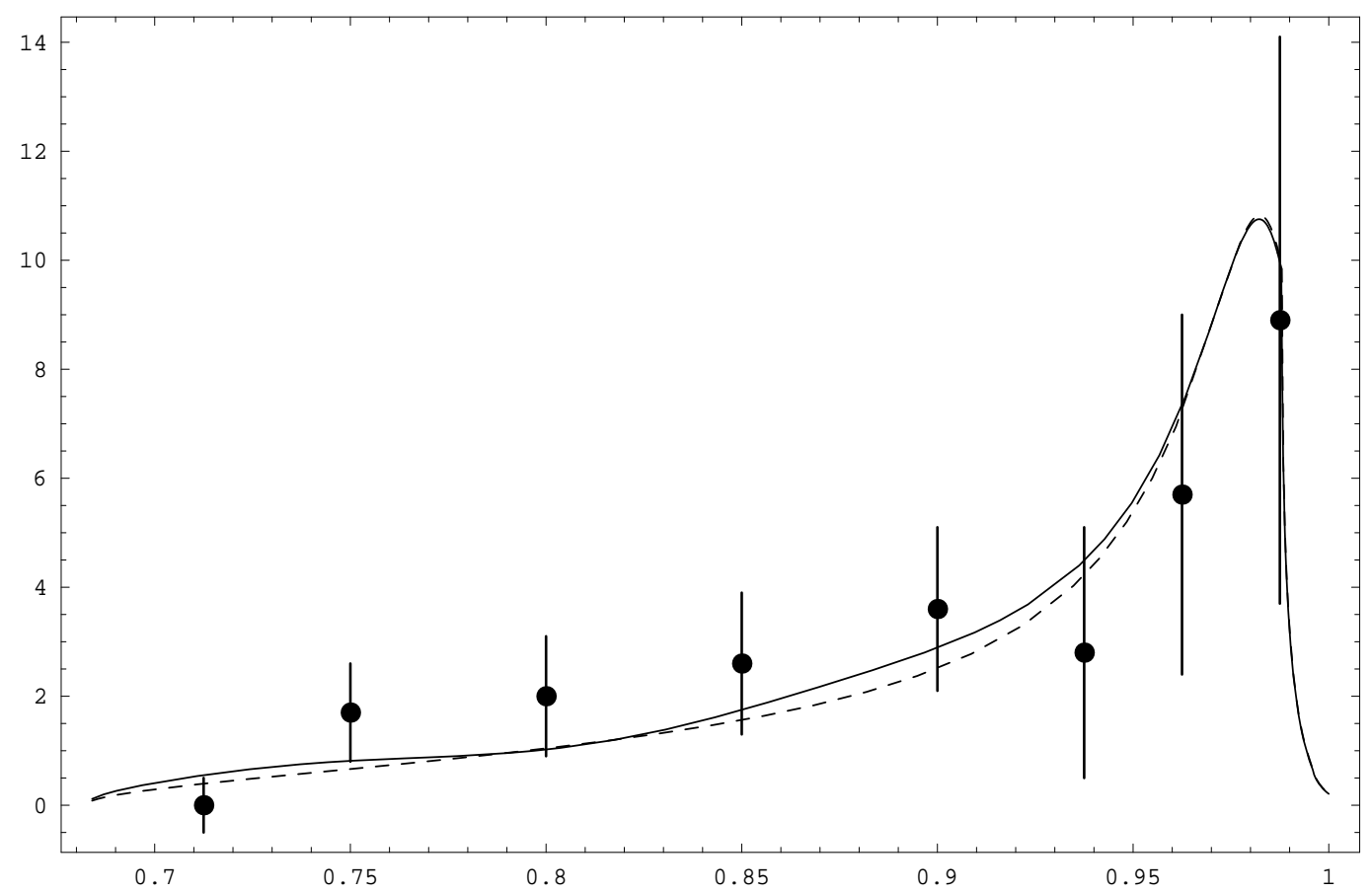

FIG. 1. Fitting of $d B R\left(\phi \rightarrow \gamma \pi^{0} \eta, m\right) / d m \times 10^{4} \mathrm{GeV}^{-1}$ is shown with the solid line, the signal contribution $\phi \rightarrow \gamma a_{0} \rightarrow \gamma \pi^{0} \eta$ is shown with the dashed line.

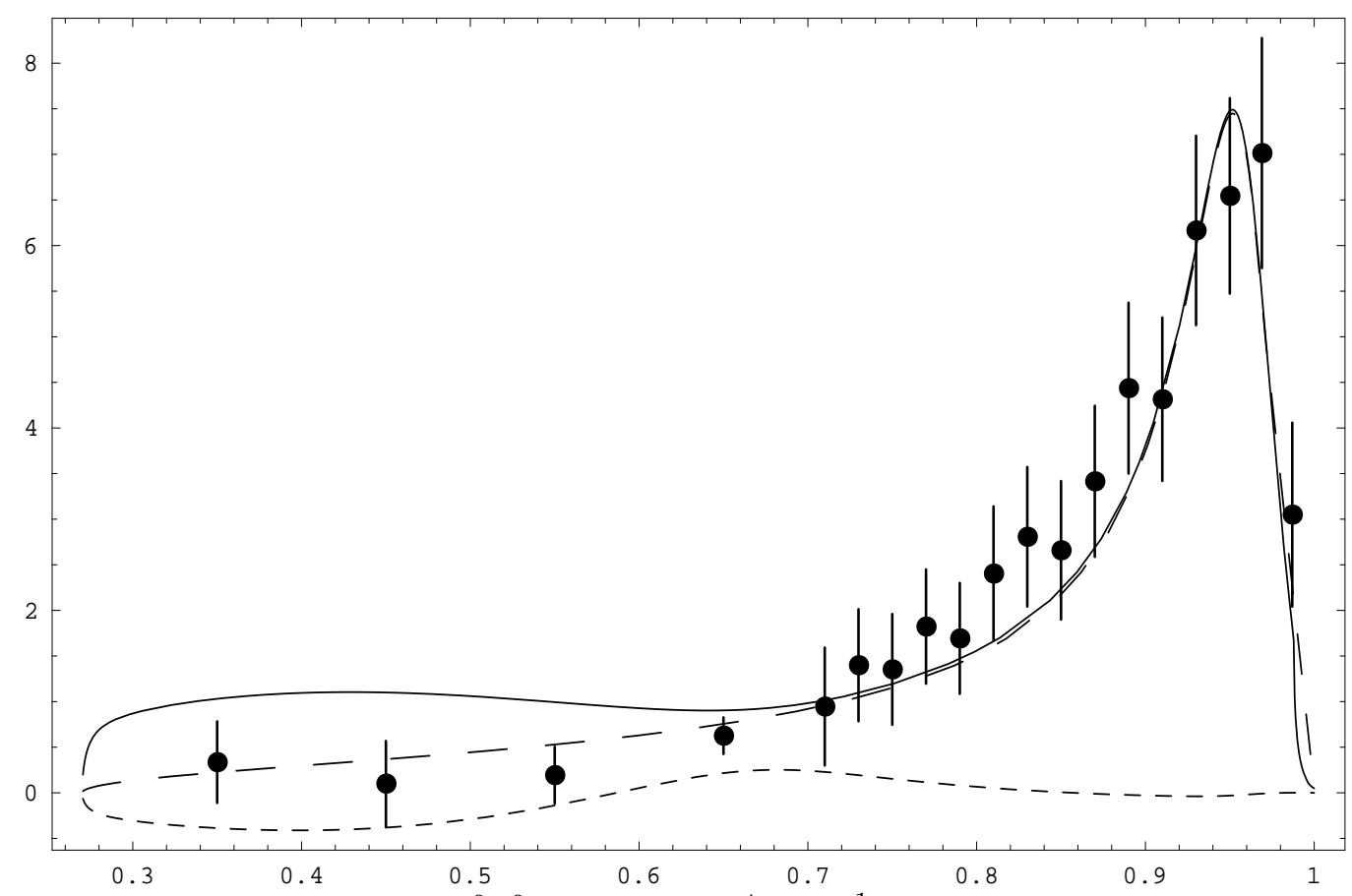

FIG. 2. Fitting of $d B R\left(\phi \rightarrow \gamma \pi^{0} \pi^{0}, m\right) / d m \times 10^{4} \mathrm{GeV}^{-1}$ is shown with the solid line, the signal contribution $\phi \rightarrow \gamma f_{0} \rightarrow \gamma \pi^{0} \pi^{0}$ is shown with the dashed line. The dots show the interference. 


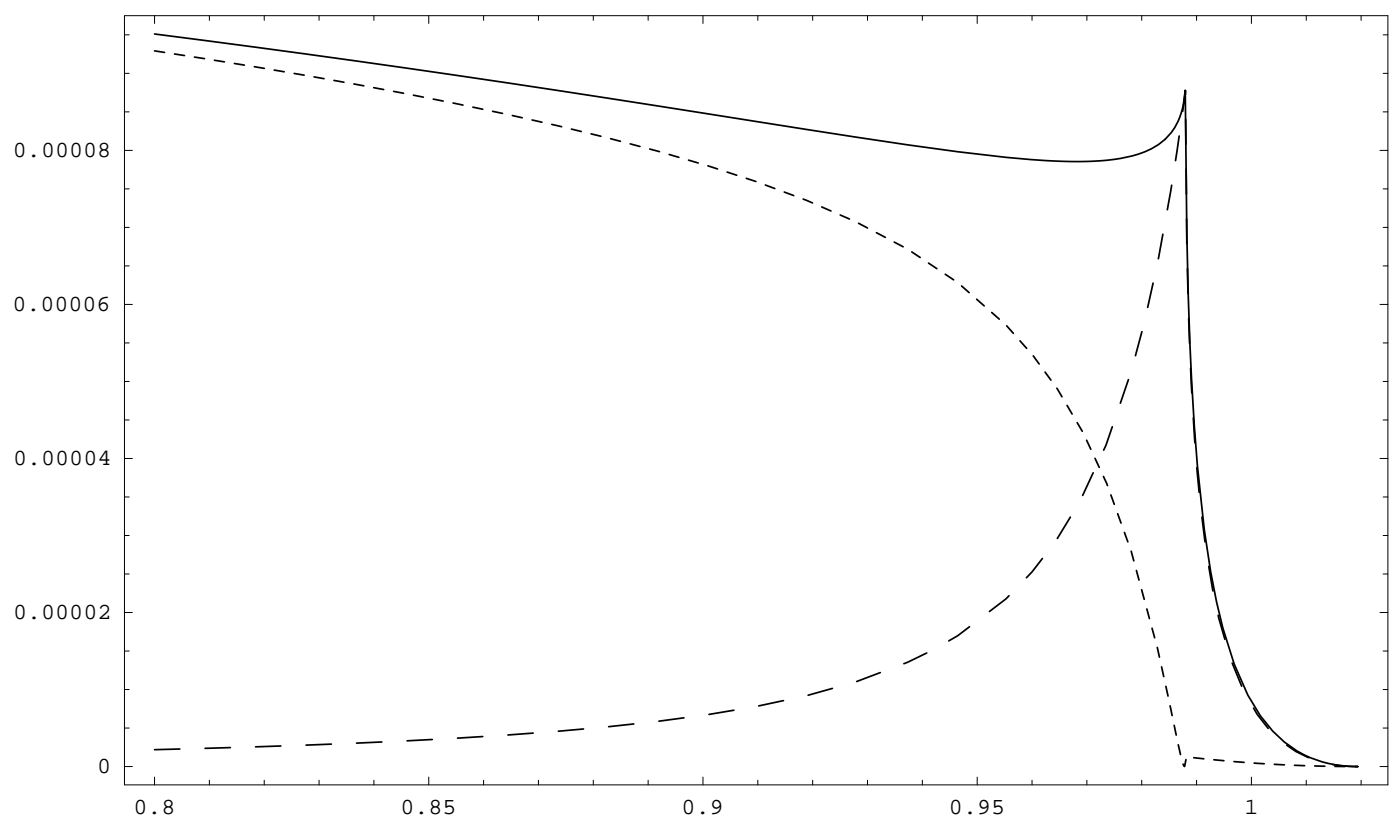

FIG. 3. The function $|g(m)|^{2}$ is drawn with the solid line. The contribution of the imaginary part is drawn with the dashed line. The contribution of the real part is drawn with the dotted line.

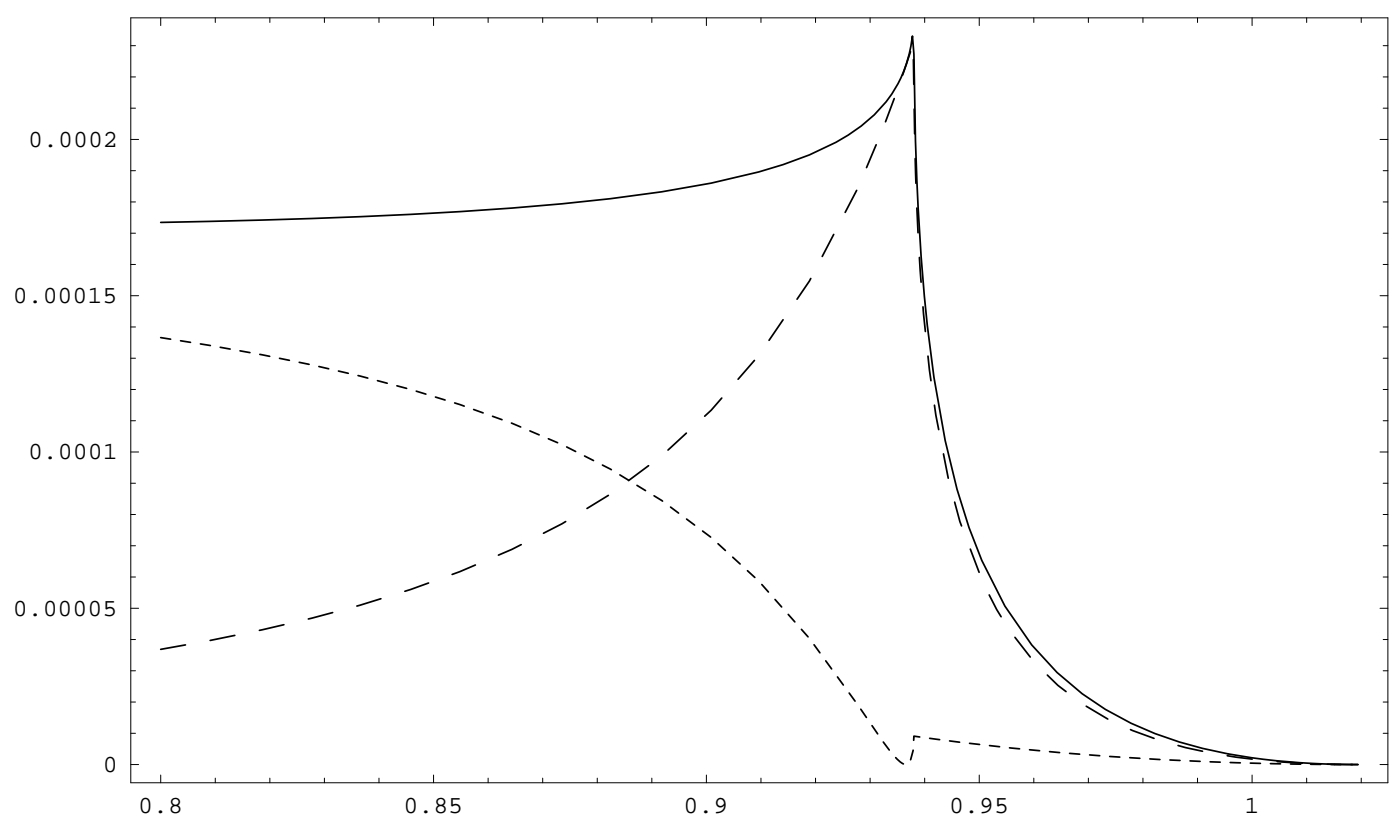

FIG. 4. The function $|g(m)|^{2}$ for $m_{K^{+}}=469 \mathrm{MeV}$ is drawn with the solid line. The contributions of the imaginary and real parts are drawn with the dashed and dotted lines respectively. 


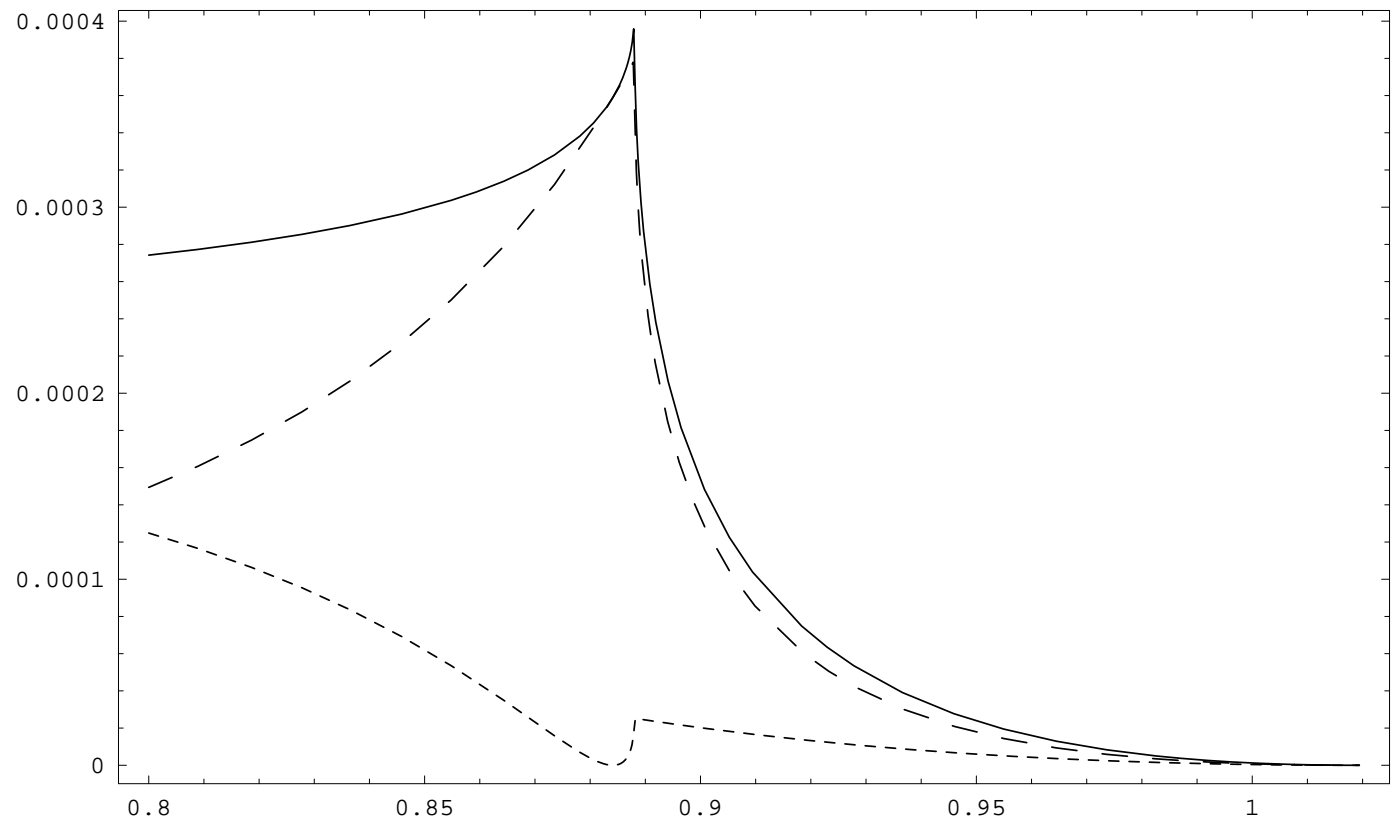

FIG. 5. The function $|g(m)|^{2}$ for $m_{K^{+}}=444 \mathrm{MeV}$ is drawn with the solid line. The contributions of the imaginary and real parts are drawn with the dashed and dotted lines respectively. 